\title{
The Impact of Signalling Kinetics on Controller Motif Performance
}

\author{
Geir B. Risvoll Tormod Drengstig \\ Dept. of Electrical Engineering and Computer Science, University of Stavanger, Norway, \\ \{geir.risvoll, tormod.drengstig\}@uis.no
}

\begin{abstract}
In this paper we investigate how the disturbance rejection properties of physiological regulatory systems depend on the signalling kinetics between the biochemical species being considered as controlled and manipulated variables. Based on the mathematical model of a physiological regulatory system, we analyse the impact of three different signalling models, i.e. linear signalling, first order and second order Hill kinetics. We separate the analysis in two parts. First we identify to what extend the signalling from the manipulated variable to the controlled variable affect the range and ratio of manageable disturbances. In the second part we investigate whether and how the signalling from the controlled variable to the manipulated variable will affect the parameters of the controller part of the regulatory system. In this context, saturable Hill kinetics display advantages compared to linear signalling.

Keywords: Systems Biology, Control Engineering, Homoeostasis, Signalling Kinetics
\end{abstract}

\section{Introduction}

In order for organisms to function properly it is imperative that their internal environment is maintained within certain thresholds independent on external conditions. This ability of living systems to adjust their own environment was first described by Claude Bernard with his definition of milieu intérieur (Cooper, 2008; Langley, 1973). Cannon later refined this concept and coined the term homeostasis (Cannon, 1929, 1939). The term homeostasis has since become the most commonly used concept when talking about an organism's ability to regulate its own internal environment in an ever changing external environment. This concept is closely related to that of control engineering, where the objective is to maintain a given setpoint, regardless of external perturbations (Skogestad and Postlethwaite, 2005).

An example of a physiological regulatory system is the regulation of cellular glucose concentration in the presence of varying blood glucose concentration. Related to the intracellular stability is also the cell's ability to counteract for e.g. large variations in intracellular consumption. For the blood glucose example, an intensive exercise session will dramatically increase the intracellular glucose consumption, leading to an increased glucose uptake from the blood stream. The physiological mechanisms behind this regulation include hormones acting as signalling species, cellular membrane receptors transferring the signalling information within the cell, and cellular membrane proteins acting as pumps. For glucose to enter a cell, the insulin hormone secreted by the pancreas is sensed by the insulin receptor located in the cell membrane. This again leads to a cascade of signalling events inside the cell, which finally activates the glucose transporter facilitating glucose uptake. In order to utilize the glucose as cellular fuel, the glucose enters the glycolysis which is a chain of enzyme catalysed reactions converting glucose into pyrovate which again is transported into the mitochondria and the TCA cycle (Jeoung and Harris, 2010; Lam et al., 2005).

The modelling of such systems involves enzymes and transporter proteins, and the models can be made at different levels of complexity, all depending on the aim of the modelling effort. If the goal is to make a detailed model of one part of the glycolysis, it can be very different compared to a model of the overall cellular homeostatic function (Ackerman et al., 1965; Swierkosz, 2015). In the latter case, the underlying model structure which would include signalling events from other cellular species is perhaps more important than details about certain enzymatic steps. In this respect, we are interested in the properties of different signalling models and how the underlying signalling kinetics will affect the performance of regulatory systems. Performance is, in this context, defined as the disturbance rejection properties of the regulatory systems, i.e. to what extend is different signalling kinetic implementations able to cope with increasing level of disturbances/perturbations. In order to compare the different signalling models we have defined a regulatory system where the involved species varies between some predefined levels. Thus, we maintain as much as possible of the surrounding cellular conditions enabling us to compare the results.

\section{Controller Motifs}

We have previously published the eight controller motifs shown in Figure 1, all being candidates for physiological regulatory systems (Drengstig et al., 2012). The function of these controller motifs show similarities with standard integral control (Drengstig et al., 2012), and their control theoretic properties have been investigated (Thorsen et al., 2013). From a synthetic biology approach, we have also shown how such controller structures can be tuned similar to an industrial control system (Thorsen et al., 2016), and 
the motifs are also shown to be closed loop asymptotically stable (Tveit and Thorsen, 2017).

The analysis in this paper is based on controller motif 5 , given in Eqs. (1) and (2).

$$
\begin{aligned}
& \dot{A}=k_{p}^{i}-V_{\text {max }}^{A} \cdot \frac{A}{K_{M}^{A}+A} \cdot f(E) \\
& \dot{E}=k_{s}^{E} \cdot g(A)-V_{\text {max }}^{E} \cdot \frac{E}{K_{M}^{E}+E}
\end{aligned}
$$

The concentrations of $A$ and $E$ represent the controlled variable and the manipulated variable, respectively. Furtermore, $k_{p}^{i}$ is the inflow perturbation and $V_{\max }^{A}$ is the maximum turnover number for $A$. The parameter $K_{M}^{A}$ is the Michaelis-Menten constant for the degradation of $A$. The synthesis rate of $E$ is given by $k_{s}^{E}$, and $V_{\max }^{E}$ is the maximum turnover number for $E$. The parameter $K_{M}^{E}$ is the Michaelis-Menten constant for the degradation of $E$. All concentrations and rate constants are given in arbitrary units (a.u.) unless stated otherwise.

The function of the motif can be described as follows. When the inflow perturbation $k_{p}^{i}$ increases, the level of $A$ increases, which again will increase the synthesis rate of $E$ through the signalling from $A$ to $E$. As the level of $E$ increases, the $E$-mediated compensatory outflow of $A$ will also increase, bringing $A$ back towards its pre-perturbed value. A necessary condition for this behaviour is that the enzyme degrading the controller species $E$ is saturated, i.e. $K_{M}^{E} \ll E$ (Drengstig et al., 2012). If this condition is not satisfied, there will be a deviation between the level of $A$ prior to and after the perturbation. Such deviation measures are found in many in silico studies of physiological regulatory systems, see e.g. (Ma et al., 2009).

The structure in Eqs. (1) and (2) is schematically illustrated in Figure 2, where the signalling from $A$ to $E$ is represented by the function $g(A)$. From a control theoretical point of view, this corresponds to the measurement in a negative feedback loop (see Figure 2.). Furthermore, the signalling from $E$ to $A$ is represented by the function $f(E)$, which is how the manipulated variable $E$ enters the process.

In our analysis we have looked further into the following three types of signalling events for both $f(E)$ and $g(A)$, commonly used to model enzyme kinetics in physiology (Cornish-Bowden, 2012) :

1. Linear signalling, i.e. $f(E)=E$ and $g(A)=A$.

2. First order Hill kinetics, i.e. $f(E)=\frac{E}{K_{a}^{E}+E}$ and $g(A)=\frac{A}{K_{a}^{A}+A}$, where $K_{a}^{E}$ and $K_{a}^{A}$ are the activation constants. The enzymatic activation is assumed to be mixed activation.

3. Second order Hill kinetics, i.e. $f(E)=\frac{E^{2}}{\left(K_{a}^{E}\right)^{2}+E^{2}}$ and $g(A)=\frac{A^{2}}{\left(K_{a}^{A}\right)^{2}+A^{2}}$

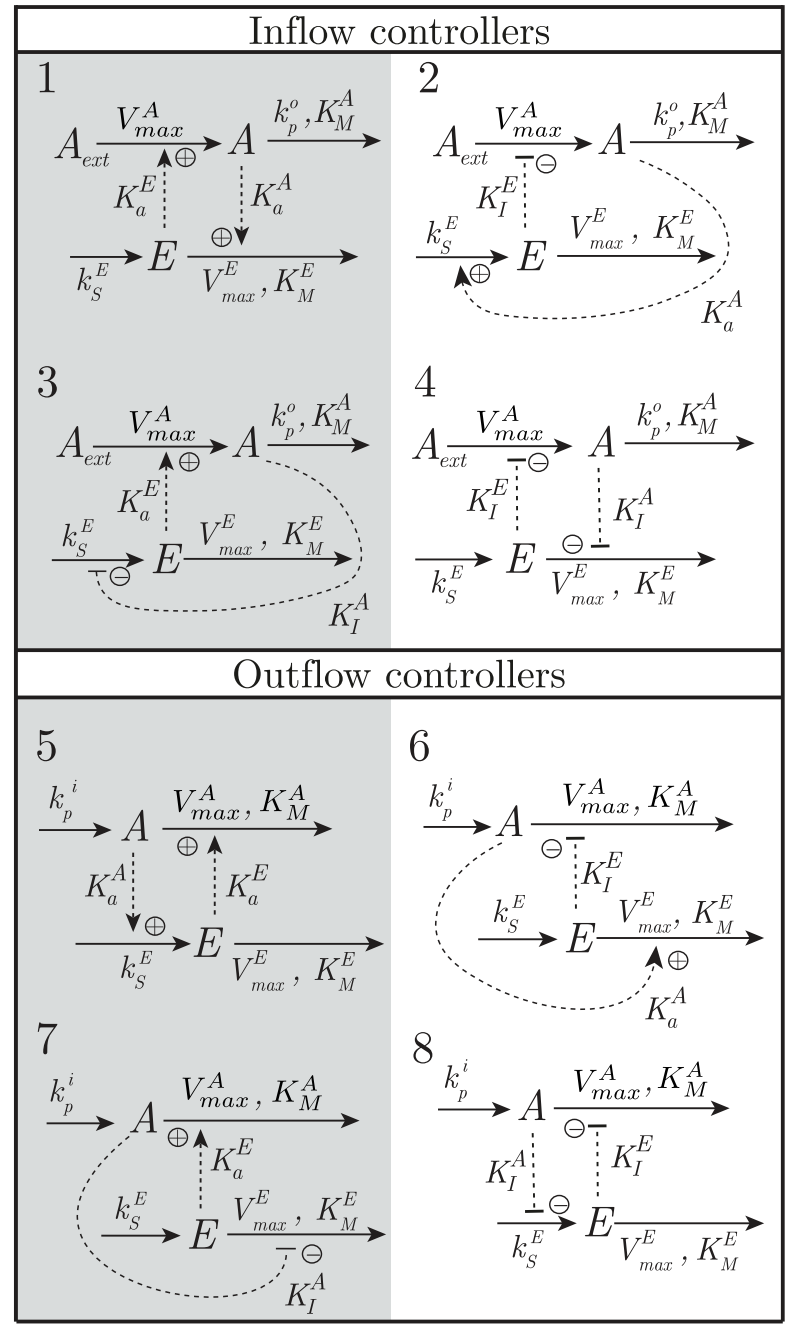

Figure 1. Eight different controller motifs, all representing negative feedback loops (Drengstig et al., 2012). Based on the location of the $E$-mediated compensatory flow, the controllers are either classified as inflow or outflow controllers. The species $A$ represent the controlled variable, and the species $E$ the manipulated variable. The perturbations are represented by the variables $k_{p}^{o}$ and $k_{p}^{i}$. The signalling between $A$ and $E$ (dashed lines) are either based on activation (plus sign) or inhibition (minus sign).

To compare these different signalling events, we have, as mentioned above, defined levels of $A$ and $E$, here represented as low and high levels, i.e.

$$
\begin{aligned}
& A \in\left[A_{\text {low }}, A_{\text {high }}\right]=[1,3] \\
& E \in\left[E_{\text {low }}, E_{\text {high }}\right]=[2,8]
\end{aligned}
$$

These levels represent combinations of steady state values of the process. The numerical values in Eqs. (3) and (4) are arbitrary chosen, and are only used to illustrate the principle.

\section{Signalling from $E$ to $A, f(E)$}

In the presence of inflow perturbation $k_{p}^{i}$, the signalling from $E$ to $A$ affect the compensatory outflow of $A$ as shown in Eq. (1). Thus, the high and low levels in Eqs. (3) and (4) 


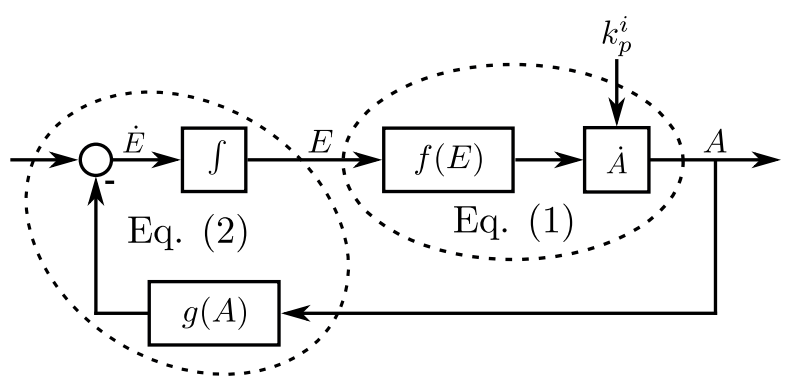

Figure 2. Representation of Eqs. (1) and (2) as a negative feedback loop. Eq. (1) represents the dynamics of the process, while Eq. (2) represents the controller dynamics.

are therefore related to corresponding high and low levels of $k_{p}^{i}$. In order to organize these 6 low/high levels into two working points, we note that a low inflow perturbation, $k_{p, l o w}^{i}$, will lead to a low level of $A$. A low level of $A$ will again lead to a low level of $E$, as $A$ activates the synthesis of $E$. Similarly, a high perturbation level $k_{p, h i g h}^{i}$, will lead to high levels of both $E$ and $A$. This behaviour is shown in the qualitative simulation shown in Figure 3, where the inflow perturbation is increased in a stepwise manner, and the corresponding levels of $A$ and $E$ are increased. Hence,

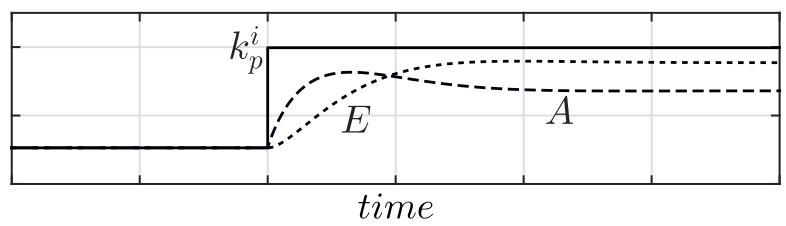

Figure 3. Qualitative simulation of motif 5. A low perturbation level $k_{p}^{i}$ results in low levels of both $E$ and $A$. Similarly, a high perturbation level $k_{p}^{i}$ results in high levels of both $E$ and $A$.

the following steady state combinations exist for motif $5^{1}$ :

$$
\begin{array}{r}
{\left[k_{p, l o w}^{i}, A_{\text {low }}, E_{\text {low }}\right]} \\
{\left[k_{p, \text { high }}^{i}, A_{\text {high }}, E_{\text {high }}\right]}
\end{array}
$$

In order to have a quantitative measure of the motif's disturbance rejection properties, we define both the range between the high and low perturbations $\left(\Delta k_{p}^{i}\right)$, and the $r a$ tio of the high to low perturbations $\left(\lambda k_{p}^{i}\right)$ as:

$$
\begin{aligned}
& \Delta k_{p}^{i}=k_{p, \text { high }}^{i}-k_{p, \text { low }}^{i} \\
& \lambda k_{p}^{i}=\frac{k_{p, \text { high }}^{i}}{k_{p, \text { low }}^{i}}
\end{aligned}
$$

Thus, our aim is to investigate how these two measures are affected by the choice of signalling event $f(E)$. The expressions for the range and ratio are found by inserting each of the steady state combinations in Eqs. (5) and (6)

${ }^{1}$ The other motifs in Figure 1 will have other combinations, determined by the kind of signalling in $f(E)$ and $g(A)$, together with the kind of controller (inflow or outflow). into Eq. (1). Thus, solving for $k_{p, l o w}^{i}$ and $k_{p, h i g h}^{i}$, respectively, we find

$$
\begin{gathered}
\Delta k_{p}^{i}=V_{\max }^{A} \frac{A_{\text {high }}}{K_{M}^{A}+A_{\text {high }}} \cdot f\left(E_{\text {high }}\right) \\
-V_{\max }^{A} \frac{A_{\text {low }}}{K_{M}^{A}+A_{\text {low }}} \cdot f\left(E_{\text {low }}\right) \\
\lambda k_{p}^{i}=\frac{\frac{A_{\text {high }}}{K_{M}^{A}+A_{\text {high }}} \cdot f\left(E_{\text {high }}\right)}{\frac{A_{\text {low }}}{K_{M}^{A}+A_{\text {low }}} \cdot f\left(E_{\text {low }}\right)}
\end{gathered}
$$

There is, in general, an important distinction between linear and Hill based signalling. While Hill kinetics are saturable, i.e. $f(E) \in\{0,1\}$, the linear signalling varies between $f(E) \in\left\{E_{\text {low }}, E_{\text {high }}\right\}$. Thus, using the same value of $V_{\max }^{A}$ would favour linear signalling with respect to range $\Delta k_{p}^{i}$. In order to make a fair comparison between linear and Hill signalling, we therefore choose $V_{\max }^{A}$ in the linear signalling case as

$$
V_{\text {max }}^{A, \text { lin }}=\frac{V_{\text {max }}^{A}}{E_{\text {high }}-E_{\text {low }}}
$$

\subsection{Linear Signalling}

In many cases, the use of linear signalling kinetics is motivated by the fact that the influence of an activator or inhibitor is not saturated, i.e. the influence is approximated to be in the linear domain of the saturation curve. As such, the $V_{\max }^{A, \text { lin }}$ is therefore far from the actual maximum level $V_{\text {max }}^{A}$, and Eq. (11) is therefore a reasonable assumption. In the following, we use $V_{\max }^{A}=1$, and hence, $V_{\max }^{A, l i n}=\frac{1}{6}$.

Linear signalling for activating kinetics is often used when modelling physiological systems (Sedaghat et al., 2002). One of the benefits is that the models are easier to analyse analytically compared to more complex signalling kinetics (Thorsen et al., 2013, 2016) .

Using $f(E)=E$ in Eqs. (9) and (10), leaves us with one unknown parameter, i.e. $K_{M}^{A}$. Thus, we present the disturbance rejection properties as a function of this parameter, see Figure 4.

We see from Figure 4a) that the range $\Delta k_{p}^{i}$ is maximized when $K_{M}^{A}=0$, and it is minimized when $K_{M}^{A} \rightarrow \infty$. At the same time, the ratio $\lambda k_{p}^{i}$ is maximized when $K_{M}^{A} \rightarrow \infty$ and minimized when $K_{M}^{A}=0$. Thus, there exists a trade-off between range and ratio in the regulatory system. Figure $4 b$ ) shows the corresponding values of $k_{p, l o w}^{i}$ and $k_{p, h i g h}^{i}$.

\subsection{First Order Hill Signalling}

The simplest saturable signalling event is the first order Hill expression:

$$
f(E)=\frac{E}{K_{a}^{E}+E}
$$


a)

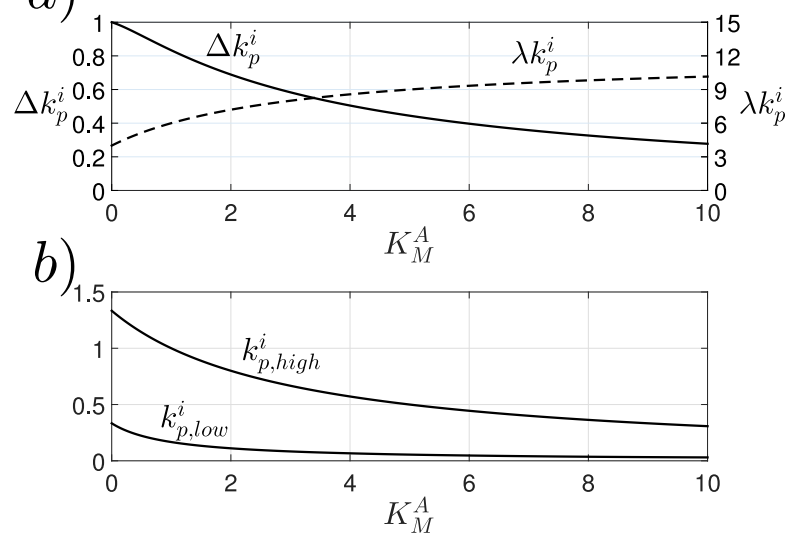

Figure 4. Panel a) The range $\Delta k_{p}^{i}$ and ratio $\lambda k_{p}^{i}$ for controller motif 5 (using $f(E)=E$ ) as a function of $K_{M}^{A}\left(V_{\max }^{A}=\frac{1}{6}\right)$. Panel b) The corresponding low and high values of $k_{p}^{i}$.

Applying Eq. (12) in Eqs. (9) and (10), gives two unknown parameters, i.e. $K_{M}^{A}$ and $K_{a}^{E}$, and thus, the range and ratio could have been presented as 3-dimensional surfaces. However, in order to focus on the effect of the signalling, we use $K_{M}^{A}=0$, and the results shown in Figure 5 are therefore comparable to the left part of Figure 4 were $K_{M}^{A}=0$.

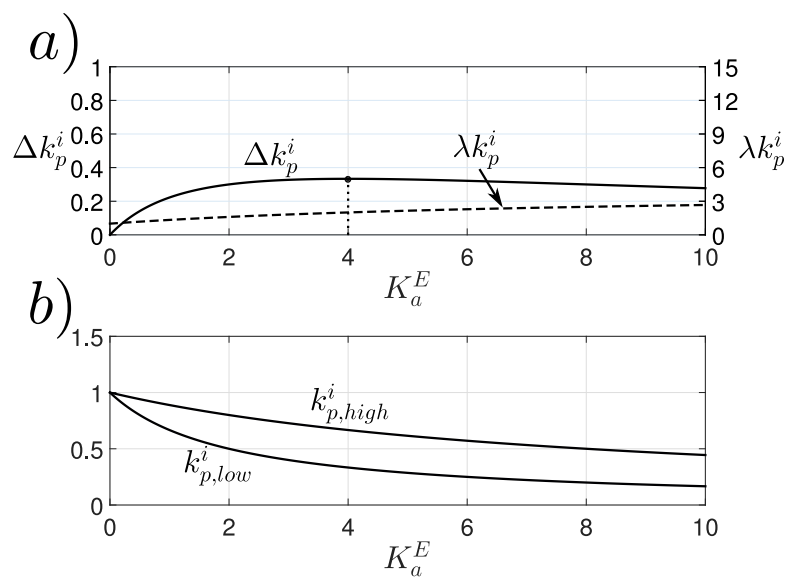

Figure 5. Panel a) The range $\Delta k_{p}^{i}$ and ratio $\lambda k_{p}^{i}$ for controller motif 5 (using $f(E)=\frac{E}{K_{a}^{E}+E}$ ) as a function of the activation constant $K_{a}^{E}\left(V_{\max }^{A}=1\right.$ and $\left.K_{M}^{A}=0\right)$. The maximum range is found at $K_{a}^{E}=4$ (indicated with a black dot). Panel b) The corresponding low and high values of $k_{p}^{i}$.

Unlike the results for the linear signalling in Figure 4a), there is a maximum value for the perturbation range. By differentiating the expression for the range $\Delta k_{p}^{i}$ (Eq. (9)) with respect to $K_{a}^{E}$, we find the maximum value as

$$
\frac{\partial \Delta k_{p}^{i}}{\partial K_{a}^{E}}=0 \quad \Rightarrow \quad K_{a}^{E}=4
$$

At $K_{a}^{E}=4$, the range is $\Delta k_{p}^{i}=0.33$ while the ratio is $\lambda k_{p}^{i}=2$.

The results indicate that the perturbation range and ratio, using first order Hill signalling from $E$ to $A$, is re- duced compared to linear signalling $\left(\Delta k_{p}^{i}=1\right.$ and $\lambda k_{p}^{i}=4$ at $K_{M}^{A}=0$ in Figure 4). It is important to note that $V_{\max }^{A}$ at the same time is increased from $V_{\max }^{A}=\frac{1}{6}$ to $V_{\max }^{A}=1$ in the Hill signalling. The decline in both perturbation range and ratio was expected when considering the Hill-based signalling. In the next section we increase the enzymatic cooperativity by increasing the order of the Hill kinetic expression.

\subsection{Second Order Hill Signalling}

The second order Hill kinetic expression is given in Eq. (14).

$$
f(E)=\frac{E^{2}}{\left(K_{a}^{E}\right)^{2}+E^{2}}
$$

As in the previous subsection, we assume $K_{M}^{A}=0$ and focus entirely on the effect of $f(E)$ through $K_{a}^{E}$, and the results are shown in Figure 6.

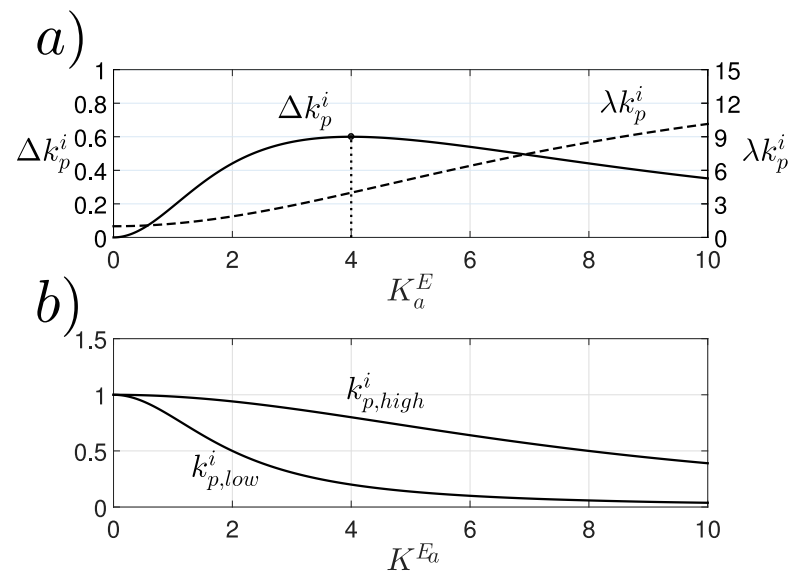

Figure 6. Panel a) The range $\Delta k_{p}^{i}$ and ratio $\lambda k_{p}^{i}$ for controller motif 5 (using $f(E)=\frac{E^{2}}{\left(K_{a}^{E}\right)^{2}+E^{2}}$ ) as a function of the activation constant $K_{a}^{E}\left(V_{\max }^{A}=1\right.$ and $\left.K_{M}^{A}=0\right)$. The maximum range is also here found at $K_{a}^{E}=4$ (indicated with a black dot). Panel b) The corresponding low and high values of $k_{p}^{i}$.

As in Figure 5a), we find in Figure 6a) a similar peak for the perturbation range at $K_{a}^{E}=4$. The noteworthy difference is that the peak is substantially higher compared to first order Hill signalling ( $\Delta k_{p}^{i}=0.6$ compared to $\Delta k_{p}^{i}=0.33$ in Figure 5a)). Moreover, the ratio $\lambda k_{p}^{i}$ is also significantly increased from 2 to 4 . Thus, both values for perturbation range and ratio for the second order Hill signalling is comparable to those from linear signalling.

\subsection{Higher Order Hill Signalling}

The general expression for higher order Hill signalling is given in Eq. (15)

$$
f(E)=\frac{E^{n}}{\left(K_{a}^{E}\right)^{n}+E^{n}}
$$

and the effect on perturbation range and ratio of increasing $n$ is shown in Figure 7. As $n$ increases, so does the per- 


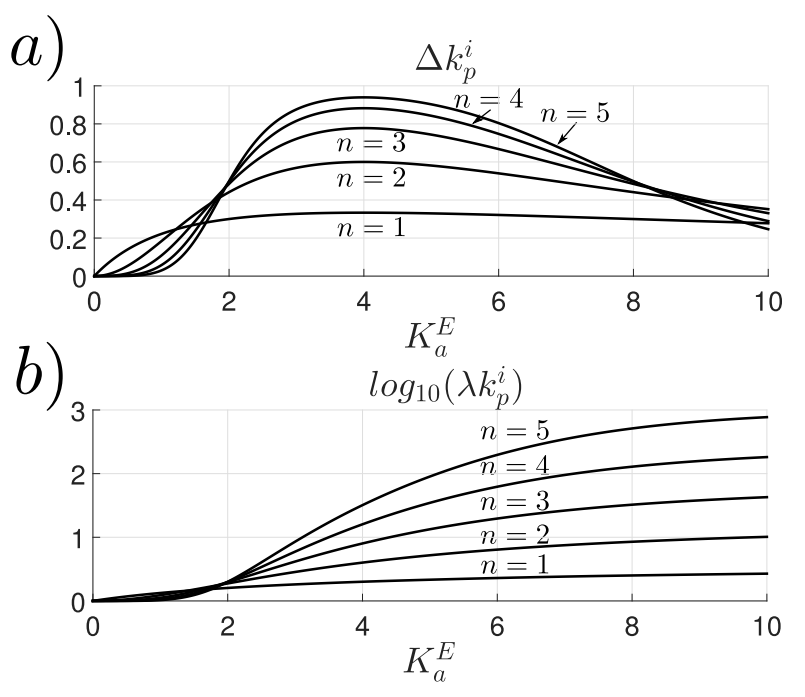

Figure 7. The range $\Delta k_{p}^{i}$ and ratio $\lambda k_{p}^{i}$ for controller motif 5 (using $f(E)=\frac{E^{n}}{\left(K_{a}^{E}\right)^{n}+E^{n}}$ for $n \in\{1 \ldots 5\}$ ) as a function of the activation constant $K_{a}^{E}\left(V_{\max }^{A}=1\right.$ and $\left.K_{M}^{A}=0\right)$. Panel a) The ranges $\Delta k_{p}^{i}$. Panel b) The ratios $\lambda k_{p}^{i}$ (note the logarithmic ordinate).

turbation range $\Delta k_{p}^{i}$ and ratio $\lambda k_{p}^{i}$. As $n \rightarrow \infty$, the range $\Delta k_{p}^{i} \rightarrow 1$ as obtained for the linear signalling.

To summarize, we have investigated the effect on the range and ratio of disturbances as function of the signalling from $E$ to $A$ given the low and high levels of $A$ and $E$ in Eqs. (3) and (4), respectively. The combination of low and high values represent therefore different possible steady state combinations (or working points) of the process.

In the next section, we investigate how the signalling $g(A)$ will affect the controller's ability to bring the process through these working points.

\section{Signalling from $A$ to $E, g(A)$}

The signalling from $A$ to $E$ represented by the function $g(A)$ is a part of the dynamic model of the controller species $E$ given in Eq. (2). Hence, the complexity of the signalling $g(A)$ will therefore, to a large degree, determine whether the steady state combinations from the previous subsection are obtainable when closing the feedback loop. As Figure 8 illustrates, the task of the controller is to match the low and high limits of $A$ and $E$, and is therefore not directly affected by perturbation levels.

The procedure in this subsection is similar to what we did in the previous subsection, i.e. we apply the signalling $g(A)$ and insert each of the two steady state combinations in Eqs. (3) and (4) into Eq. (2). Thus, we have two equations and three unknowns i.e. $k_{s}^{E}, V_{\max }^{E}$, and $K_{M}^{E}$. For the cases where we apply Hill based signalling, we have an addition unknown parameter in the activation constant $K_{a}^{E}$.

In general, the level of the synthesis rate $k_{s}^{E}$ and degradation rate $V_{\max }^{E}$ of $E$ is a measure of the swiftness of the controller (Thorsen et al., 2016). These two parameters are therefore individually not a part of the steady

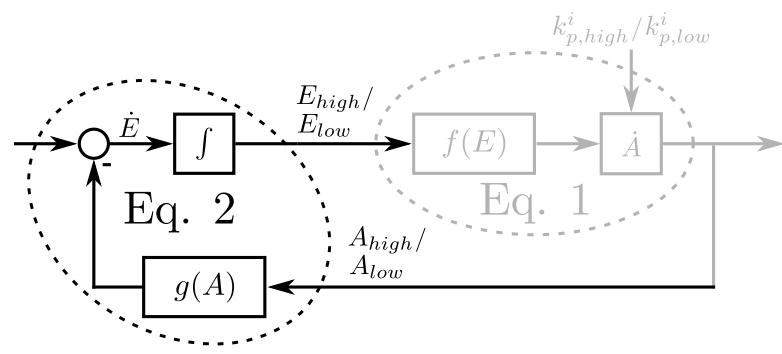

Figure 8. An illustration showing that the controller part is only dependent on the low and high levels of $E$ and $A$, and indirectly dependent on the perturbation levels.

state properties of the controller. However, their ratio participate in the definition of the physiological setpoint (Thorsen et al., 2016), which represents the steady state levels of $E$. In this subsection, we therefore specify one of the parameters, i.e. $V_{\max }^{E}=1$, and calculate the other.

\subsection{Linear Signalling}

In case of linear signalling, we have $g(A)=A$. The two unknown parameters $K_{M}^{E}$ and $k_{s}^{E}$ are calculated by solving the two versions of Eq. (2). Interestingly, in order to find positive solutions to these parameter, the following condition on the steady state levels must be satisfied

$$
\frac{E_{\text {high }}}{E_{\text {low }}}>\frac{A_{\text {high }}}{A_{\text {low }}}
$$

Thus, if the variation in the controlled variable $A$ is too large compared to the variation in the manipulated variable $E$, the controller is not able to bring the process through the identified working points using linear activation.

\subsection{First Order Hill Signalling}

If we apply first order Hill signalling from $A$ to $E$, we have

$$
g(A)=\frac{A}{K_{a}^{A}+A}
$$

One could expect that this saturable expression would complicate the process of finding a suitable controller even further, i.e. introducing stricter conditions compared to Eq. (16). However, to our surprise, the signalling in Eq. (17) enables us to identify controllers where Eq. (16) is not fulfilled. Thus, if the low and high steady state levels of $E$ and $A$ do not satisfy Eq. (16), the following additional condition on $K_{a}^{A}$

$$
K_{a}^{A}<\frac{A_{\text {high }} \cdot A_{\text {low }} \cdot\left(E_{\text {high }}-E_{\text {low }}\right)}{A_{\text {high }} \cdot E_{\text {low }}-A_{\text {low }} \cdot E_{\text {high }}}
$$

will make the control system feasible. The expression in Eq. (18) is found in the symbolic expression for the solution to $K_{M}^{E}$ and $k_{s}^{E}$ when solving the two versions of Eq. (2). 
In order to illustrate this phenomena, we show in Figure 9 three different simulations where

$$
\begin{aligned}
& A \in\left[A_{\text {low }}, A_{\text {high }}\right]=[1,3] \\
& E \in\left[E_{\text {low }}, E_{\text {high }}\right]=[2, \mathbf{5}]
\end{aligned}
$$

Note that we have reduced $E_{\text {high }}=5$, and hence, the condition in Eq. (16) is therefore not satisfied. The signalling $f(E)$ from $E$ to $A$ is in all three simulations based on the first order Hill kinetics in Eq. (12). Thus, using the new low and high levels in Eqs. (19) and (20), the optimum $K_{a}^{E}$ is found to be $K_{a}^{E}=3.16$.

For the linear signalling $g(A)=A$, we get the dashed lines in Figure 9. We note that, as expected, the controller is not able to bring the process through the working points. The solid line in Figure 9 represents the first order Hill signalling in $g(A)$ where the constraint on $K_{a}^{A}$ in Eq. (18) is met, i.e. $K_{a}^{A}=5<9$. In order to show the effect of not satisfying either Eq. (16) nor Eq. (18), we have included this simulation as dotted lines as well. Both parameters $K_{M}^{E}$ and $k_{s}^{E}$ are kept constant for all three simulations. In order to avoid negative values of $A$, we have used $K_{M}^{A}=0.001$.

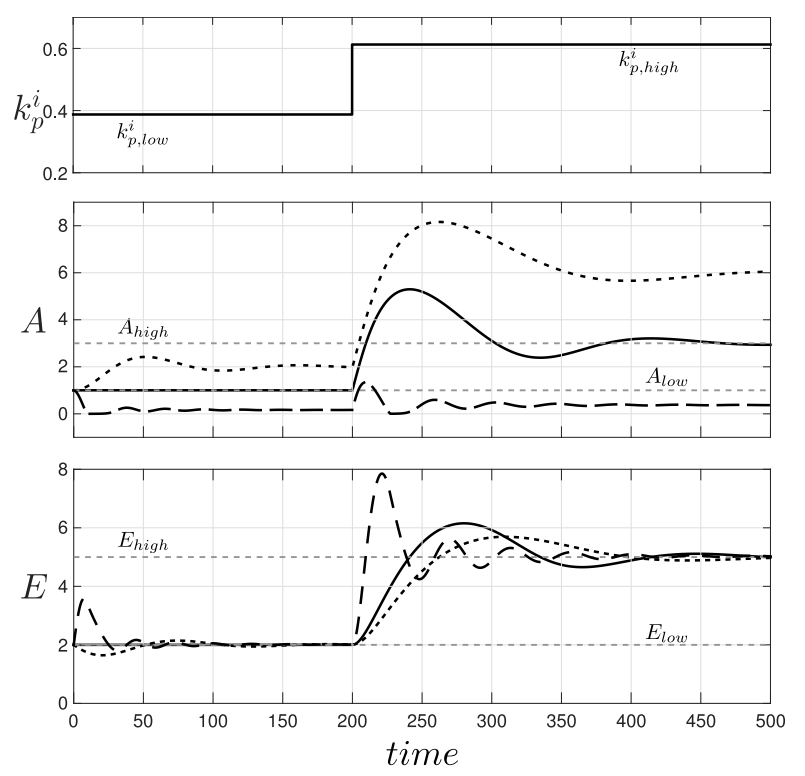

Figure 9. Simulations of controller motif 5 using three different signalling events between $A$ and $E$, constrained by the steady state levels in Eqs. (19) and (20). Solid lines: First order Hill signalling using $K_{a}^{A}=5$, satisfying the condition in Eq. (18). Dotted lines: First order Hill signalling using $K_{a}^{A}=10$, violating the condition in Eq. (18). Dashed lines: Linear signalling. Other parameter values used in these simulations are: $K_{M}^{A}=0.001, K_{a}^{E}=3.16, V_{\max }^{E}=1, k_{s}^{E}=0.44$ and $K_{M}^{E}=25$.

\subsection{Second Order Hill Signalling}

Extending the Hill signalling to second order, we have

$$
g(A)=\frac{A^{2}}{\left(K_{a}^{A}\right)^{2}+A^{2}}
$$

Similar to the above results, we find conditions on both i) the values of steady state levels (similar to Eq. (16)) and $i i)$ the parameter $K_{a}^{A}$ (similar to Eq. (18)). These new conditions are shown in Eqs. (22) and (23).

$$
\begin{gathered}
\frac{E_{\text {high }}}{E_{\text {low }}}>\frac{A_{\text {high }}^{2}}{A_{\text {low }}^{2}} \\
K_{a}^{A}<\frac{\sqrt{\left(E_{\text {high }}-E_{\text {low }}\right)\left(E_{\text {low }} A_{\text {high }}^{2}-E_{\text {high }} A_{\text {low }}^{2}\right)}}{E_{\text {low }} A_{\text {high }} A_{\text {low }}^{-1}-E_{\text {high }} A_{\text {low }} A_{\text {high }}^{-1}}
\end{gathered}
$$

We observe that the condition in Eq. (22) is stricter compared to Eq. (16). Moreover, inserting the steady state levels in Eqs. (19) and (20) into Eq. (23) reveals that also this condition is strengthened compared to first order Hill signalling.

\section{Conclusions}

We have in this paper investigated the effects of different signalling kinetics between the controlled and manipulated variables in physiological regulatory systems. The results indicate that the level of complexity in the signalling certainly has an influence on the disturbance rejection properties.

By introducing first order Hill signalling from $E$ to $A$ via the function $f(E)$, we initially experience a drop in performance in both range $\left(\Delta k_{p}^{i}\right)$ and ratio $\left(\lambda k_{p}^{i}\right)$. However if we allow second order Hill signalling the performance becomes comparable to the linear case, and by allowing higher order Hill signalling we find that the ratio $\Delta k_{p}^{i}$ is significantly improved over linear signalling.

In the signalling from $A$ to $E$, represented by the function $g(A)$, we find that the introduction of Hill signalling allows us to model data which would not be possible when using linear signalling.

\section{References}

E. Ackerman, L. C. Gatewood, J. W. Rosevear, and G. D. Molnar. Model studies of blood-glucose regulation. The Bulletin of Mathematical Biophysics, 27:21-37, 1965. doi:10.1007/BF02477259.

W. B. Cannon. Organization for physiological homeostasis. Physiological Reviews, 9(3):399-431, 1929.

W. B. Cannon. The Wisdom of the Body. Revised and Enlarged Edition. Norton, New York, 1939. ISBN 978-0393002058.

S. J. Cooper. From Claude Bernard to Walter Cannon. Emergence of the concept of homeostasis. Appetite, 51(3):419427, 2008. doi:10.1016/j.appet.2008.06.005.

A. Cornish-Bowden. Fundamentals of Enzyme Kinetics, 4th Edition. Wiley-Blackwell, 2012. ISBN 978-3-527-33074-4.

T. Drengstig, I. W. Jolma, X. Y. Ni, K. Thorsen, X. M. $\mathrm{Xu}$, and P. Ruoff. A basic set of homeostatic controller motifs. Biophysical Journal, 103(9):2000-2010, 2012. doi:10.1016/j.bpj.2012.09.033. 
N. H. Jeoung and R. A. Harris. Role of pyruvate dehydrogenase kinase 4 in regulation of blood glucose levels. Korean Diabetes Journal, 34(5):274-283, 2010. doi:10.4093/kdj.2010.34.5.274.

T. K. T. Lam, R. Gutierrez-Juarez, A. Pocai, and L. Rossetti. Regulation of blood glucose by hypothalamic pyruvate metabolism. Science, 309(5736):943-947, 2005. doi: $10.1126 /$ science. 1112085 .

L. L. Langley. Homeostasis: Origins of the Concept. Dowden, Hutchinson \& Ross, 1973. ISBN 9780879330071.

W. Ma, A. Trusina, H. El-Samad, W. A. Lim, and C. Tang. Defining network topologies that can achieve biochemical adaptation. Cell, 138(4):760-773, 2009. doi:10.1016/j.cell.2009.06.013.

A. R. Sedaghat, A. Sherman, and M. J. Quon. A mathematical model of metabolic insulin signaling pathways. American Journal of Physiology - Endocrinology And Metabolism, 283(5):E1084-E1101, nov 2002. doi:10.1152/ajpendo.00571.2001.

S. Skogestad and I. Postlethwaite. Multivariable Feedback Control. Wiley, 2005. ISBN 978-0470011683.

A. Swierkosz. Modeling of metabolic diseases - a review of selected methods. Bio-Algorithms and Med-Systems, 11(4): 205-209, jan 2015. doi:10.1515/bams-2015-0016.

K. Thorsen, P. Ruoff, and T. Drengstig. Control theoretic properties of physiological controller motifs. In 2013 International Conference on System Science and Engineering (ICSSE), pages 165-170. IEEE, jul 2013. doi:10.1109/ICSSE.2013.6614653.

K. Thorsen, G. B. Risvoll, D. M. Tveit, P. Ruoff, and T. Drengstig. Tuning of physiological controller motifs. In 9th EUROSIM Congress on Modelling and Simulation, pages 28-33, Oulu, 2016. ISBN 978-1-5090-4119-0.

D. M. Tveit and K. Thorsen. Passivity-based analysis of biochemical networks displaying homeostasis. To be presented at SIMS 2017, 2017. 